\title{
ПРОСТРАНСТВЕННО-КООРДИНАТНОЕ ОБЕСПЕЧЕНИЕ ВЕДЕНИЯ 3D-КАДАСТРА В РОССИИ
}

\section{Евгений Ильич Аврунев}

Сибирский государственный университет геосистем и технологий, 630108, Россия, г. Новосибирск, ул. Плахотного, 10, кандидат технических наук, директор Института кадастра и природопользования, тел. (383)344-31-73, e-mail: kadastr204@yandex.ru

\section{Артур Ильгизович Гиниятов}

Сибирский государственный университет геосистем и технологий, 630108, Россия, г. Новосибирск, ул. Плахотного, 10, аспирант, тел. (383)344-31-73, e-mail: ita1095@mail.ru

В работе рассматриваются основные положения создания геодезического обоснования (ГО) для ведения 3D-кадастра в России. В соответствии с ними создание ГО может быть представлено в виде двух технологических процессов: построения ГО в кадастровом квартале для координирования характерных точек контуров объектов капитального строительства (ОКС) и 3D-моделирования внутренних элементов (помещений) ОКС.

Для выполнения геодезических измерений, построения системы координат и выполнения 3D-моделирования структурных элементов ОКС предложена соответствующая нормативная база. Реализация предложенных технологических решений позволяет рассматривать технический план, формируемый кадастровым инженером, не только как документ, необходимый для постановки ОКС и его структурных элементов на государственный кадастровый учет (ГКУ), но и как нормативно-правовой документ, определяющий соответствие возведенного ОКС своим проектным параметрам.

Ключевые слова: объект недвижимости, Единый государственный реестр недвижимости, государственный кадастровый учет, 3D-кадастр, 3D-модель, объект капитального строительства, характерная точка, координаты, средняя квадратическая ошибка, нормативная точность

\section{GEODETIC SUPPORT OF 3D CADASTRE MANAGEMENT IN RUSSIA}

\section{Evgeny I. Avrunev}

Siberian State University of Geosystems and Technologies, 10, Plakhotnogo St., Novosibirsk, 630108, Russia, Ph. D., Director, Institute of Cadastre and Environmental Management, phone: (383)344-31-73, e-mail: kadastr204@yandex.ru

\section{Artur I. Giniyatov}

Siberian State University of Geosystems and Technologies, 10, Plakhotnogo St., Novosibirsk, 630108, Russia, Ph. D. Student, phone: (383)344-31-73, e-mail: ita1095@mail.ru

The paper considers the main provisions for creating a geodetic justification (GJ) for maintaining a 3D-cadastre in Russia. In accordance with them, the creation of GJ can be represented in the form of two technological processes: construction of the GJ in the cadastral quarter to coordinate the characteristic points of the contours of capital construction objects (CCO) and 3Dmodeling of internal elements of the CCO.

A corresponding regulatory framework is proposed for performing geodetic measurements, constructing a coordinate system, and performing 3D modeling of structural elements of the CCO. 
The implementation of the proposed technological solutions allows considering the technical plan formed by the cadastral engineer, not only as a document necessary for setting the $\mathrm{CCO}$ and its structural elements on the State Cadastral Register (SCR), but also as a regulatory document that determines the compliance of the constructed $\mathrm{CCO}$ with its design parameters.

Keywords: estate object, Unified State Register of Real Estate, State Cadastral Registration, 3D cadastre, 3D-model, object of capital construction, characteristic point, coordinates, root mean square error, standard accuracy

Характерной особенностью развития кадастра недвижимости в нашей стране в последнее десятилетие являются постоянно расширяющиеся, как по объемам, так и по существу рассматриваемых вопросов, исследования и разработки в области создания и ведения трехмерного кадастра (3D-кадастра) [1-5].

Анализ современного состояния проблемы создания и ведения 3Dкадастра как в России, так и за рубежом, позволил сделать некоторые заслуживающие, на наш взгляд, особого внимания выводы, существо которых заключается в следующем [1].

На сегодняшний день для формирования 3D-кадастра в зарубежных странах характерна четко прослеживаемая тенденция внедрения BIM (Building Information Model) - технологий при формировании объектов недвижимости (OH), представленных в массе своей объектами капитального строительства (OKC).

Создание нового 3D-кадастра осуществляется на основе существующего 2D-кадастра, для чего повсеместно используется бюджетная форма конвертации данных с параллельным внедрением BIM-технологий на этапе проектирования новых ОКС [6].

В результате развития современных технологий в области трехмерного моделирования на достаточно высоком уровне решена задача визуализации описания наземных ОН, когда существующие сервисы создают их качественные трехмерные модели, чего пока нельзя сказать о подземных $\mathrm{OH}$.

При всем при этом, о точности определения местоположения характерных точек $\mathrm{OH}$ и о пространственной привязке $\mathrm{OH}$, в целом, речи не идет. Все рассуждения идут вокруг точности моделирования, которая на сегодняшний день характеризуется миллиметровыми величинами [7].

В данной работе авторы хотели бы довести до читателя свою концепцию пространственно-координатного обеспечения создания и ведения 3D-кадастра, в первую очередь, и, в некоторой степени, создания 3D-кадастра, в целом, применительно к условиям России. Для этого будет рассмотрено несколько аспектов, определяющих конкретные особенности нашего государства и одновременно являющиеся факторами, влияющими на решение поставленных задач.

В первую очередь, отметим весьма существенные размеры территории Российской Федерации. Нелишне будет вспомнить, что земельный фонд России занимает первое место в мире тогда, как большинство государств, где создан и функционирует трехмерный кадастр $[6,8,9]$, по своим размерам сопоставимы с территориями субъектов РФ. А ведь это весьма важный аспект, предполагающий большую трудоемкость выполняемых технологических операций при создании 3D-кадастра в условиях обширных территорий Российской Федерации и, соответственно, значительные финансовые затраты. 
Учитывая обширность территории России и количество $\mathrm{OH}$, расположенных на ней и подлежащих ГКУ, нетрудно понять, что задача создания и ведения 3D-кадастра представляет собой весьма и весьма сложную проблему, которую можно сравнить разве, что с созданием государственной геодезической сети на территории бывшего Советского Союза, длившемся несколько десятилетий. В связи с этим представляется, что кадастр недвижимости в нашей стране должен быть комбинированным. Иными словами, часть сведений кадастра должна оставаться в прежнем виде (2D-кадастр), а часть его, там, где существует объективная необходимость, должна быть трансформирована в 3D-кадастр.

Следующий аспект, который необходимо отметить, заключается в том. что ведение 2D-кадастра и градостроительное проектирование, как правило, осуществляется в разных координатных системах, что в ряде случаев (при различном удалении осевого меридиана от центральной части территориального образования) обусловливает систематические расхождения между проектными размерами ОКС и их значениями, полученными в результате координирования относительно исходных пунктов межевого съемочного обоснования (МСО) [5].

И, наконец, еще один важный аспект, касающийся введения третьей координаты, определяющей местоположение характерных точек границ (контуров) $\mathrm{OH}$. Здесь нужно отметить, что в действующей нормативно-правовой литератуpe, регламентирующей требования к точности выполнения кадастровых работ, отсутствуют научно-обоснованные требования к средним квадратическим ошибкам (СКО) построения геодезического обоснования, необходимого при выполнении кадастровых работ для получения пространственных данных о местоположении ОН в соответствующем территориальном образовании.

Таким образом, подытоживая вышеизложенное, можно сделать вывод, что основная проблематика создания и ведения $3 \mathrm{D}$-кадастра в нашей стране на современном этапе обусловлена отсутствием нормативных требований к точности определения пространственных данных при координировании ОКС и применяемой при математической обработке результатов измерений координатной системы. Решение этой важной научно-технической проблемы позволит создать единое геопространство территориального образования, необходимое для решения многочисленных задач для устойчивого развития территорий и наполнить Единый государственный реестр недвижимости (ЕГРН) достоверной и актуальной информацией о пространственном местоположении ОКС [10-13].

Алгоритм решения проблемы должен включать в себя реализацию следующих основных методических решений.

Bo-первых, создание 3D-кадастра наименее трудоемко и более технологично осуществлять на базе существующего 2D-кадастра и в соответствии с требованиями, установленными действующими, на сегодняшний день, нормативно-правовыми документами. При этом для координирования ОН следует использовать местную плоскую прямоугольную систему координат, используемую в настоящее время для ведения 2D-кадастра.

Во-вторых, для устранения системных противоречий между результатами координирования ОКС (при выполнении кадастровых работ в их отношении) и их проектными значениями, которые должны соответствовать размерам на 
физической поверхности Земли, целесообразно при выполнении 3Dмоделирования использовать местную систему координат ОКС, привязанную к его основным осям и координатной системе территориального образования.

И, в-третьих, предлагается рассматривать кадастровый квартал как структурную единицу территориального образования, для которой необходимо установить свои научно-обоснованные нормативные требования на точность выполнения кадастровых работ.

Как было отмечено выше, технологичность перевода действующего кадастра недвижимости в 3D-кадастр определяет целесообразность использования существующей плоской прямоугольной координатной системы территориального образования с добавлением третьей координаты - нормальной высоты $\mathrm{H}$, для которой необходимо установить соответствующий нормативный допуск.

Предлагается установить нормативную точность определения высотной составляющей пространственной характеристики ОКС исходя из принципа «изотропности» реального геопространства, определяющего одинаковую значимость пространственных координат ОН на плоскости и по высоте. Помимо этого, исходя из предлагаемых концептуальных положений и целесообразности создания 3D-кадастра на основании существующей нормативно-правовой базы представляется целесообразным поддержать предложения сделанные в работе [14] об установлении нормативного допуска в плановом положении на среднюю квадратическую ошибку (СКО) взаимного положения характерных точек контуров ОКС внутри кадастрового квартала $\mathrm{m}_{\mathrm{OKC}}=5 \mathrm{~cm}$. Установление такого допуска, во-первых, позволит с заданной нормативной точностью $\left(\mathrm{m}_{3 \mathrm{y}}=10 \mathrm{~cm}\right)$ восстанавливать на местности утраченные границы земельных участков, а, вовторых, сохранит плановую составляющую существующего кадастра для наиболее технологичного перевода его в формат 3D.

Тогда с учетом всего вышесказанного можем записать:

$$
m_{x, y}=\sqrt{m_{x}^{2}+m_{y}^{2}}=m_{H}=5 \mathrm{cM} ;
$$

Как следствие, нормативная СКО пространственного положения характерных точек контуров ОКС внутри кадастрового квартала составит:

$$
m_{\text {OKC }}=\sqrt{m_{x, y}^{2}+m_{H}^{2}}=\sqrt{5_{c M}^{2}+5_{c M}^{2}}=7,1 c M ;
$$

В соответствии с установленным нормативом (1) и (2) определим нормативные требования к точности построения внутри кадастрового квартала межевого съемочного обоснования (МСO):

$$
m_{M C O}=\frac{m_{O K C}}{t}=\frac{\sqrt{m_{x, y}^{2}+m_{H}^{2}}}{t}=\frac{\sqrt{5_{c u}^{2}+5_{c u}^{2}}}{2}=3,6 \mathrm{~cm} .,
$$

где величина $t$ представляет собой статистический коэффициент, зависящий от доверительной вероятности $\mathrm{P}(\mathrm{P}=95 \%-\mathrm{t}=2 ; \mathrm{P}=99 \%-\mathrm{t}=2,5 ; \mathrm{P}=99,73 \%-\mathrm{t}=$ $3)$, в нашем случае рекомендуется $\mathrm{t}=2$. 
Обзор существующей нормативно-правовой базы формирования технических планов и выполнения исполнительных съемок при приеме ОКС в эксплуатацию показал, что точность геодезических измерений, в зависимости от размеров помещения (длины и ширины), составляет от 2 до 10 мм [15]. Таким образом, СКО определения параметров внутренних структурных элементов ОКС является более высокой по сравнению с внешним контуром, который координируется относительно исходных пунктов МСО.

Следовательно, использование при выполнении 3D-моделирования координатной системы ЕГРН приведет к искаженным результатам и не позволит оценивать соответствие параметров возведенного ОКС проектным значениям.

Выход из создавшейся ситуации, по нашему мнению, заключается в следующем: при 3D-моделировании ОКС необходимо использовать условную систему координат ОКС, привязанную к координатной системе ЕГРН одной характерной точкой (XТ), координаты которой определены относительно пунктов МСО.

Построение такой условной координатной системы ОКС предлагается осуществлять путем совмещения координатных осей с его основными осями, а за начало системы координат принимать ХТ ОКС, закоординированную относительно пунктов МСО. Принципиальная схема такого подхода приведена на рисунке.

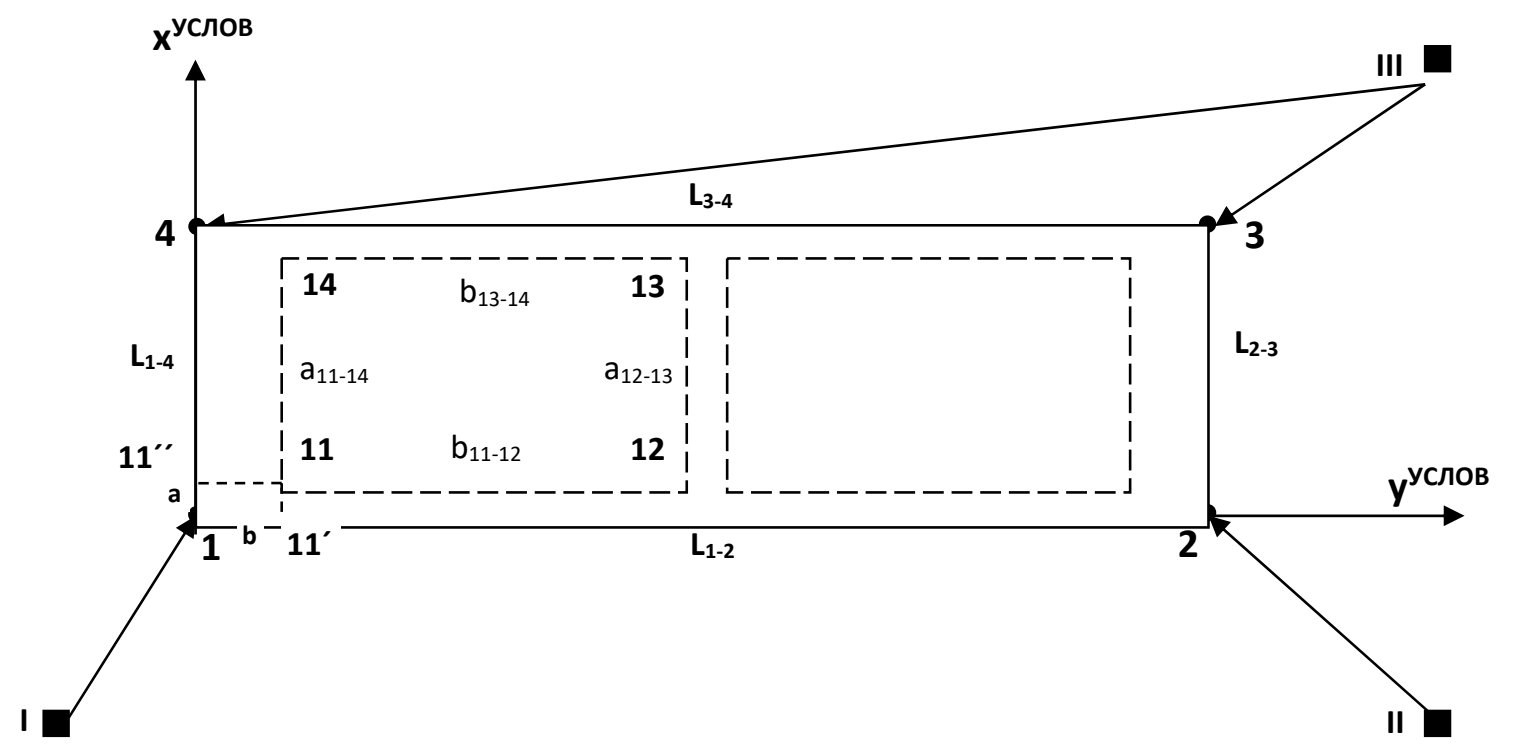

Принципиальная схема определения пространственных характеристик ОКС для 3D-моделирования и внесения этих данных в ЕГРН: где $\mathrm{a}, \mathrm{b}$ - проектные расстояния, определяющие расположение основных и дополнительных осей; $\mathrm{a}_{\mathrm{i}-\mathrm{j}}, \mathrm{b}_{\mathrm{i}-}$ j- измеренные параметры внутренних помещений ОКС; 11, 12, 13, 14 - характерные точки, определяющие параметры внутренних структурных элементов ОКС и его дополнительных осей; $1,2,3,4$ - характерные точки, определяющие расположение основных осей ОКС; I, II, III - пункты МСО. 
Реализация такого подхода позволит решить следующие научнотехнические задачи:

1) использовать технический план, формируемый кадастровым инженером в отношении ОКС, как независимый контроль при приеме в эксплуатацию построенного инженерного сооружения;

2) восстанавливать утраченные границы земельных участков вне зависимости от меняющихся систем координат и исходных пунктов геодезического обоснования, используемых при осуществлении кадастровых работ;

3) выбирать схемы построения МСО и используемое при этом измерительное технологическое оборудование для получения нормативной точности пространственного положения ОКС в кадастровом квартале соответствующего территориального образования;

4) перевести существующий 2D-кадастр недвижимости в 3D-формат с минимальными финансовыми затратами, что весьма актуально на современном этапе развития земельно-имущественных отношений.

\section{БИБЛИОГРАФИЧЕСКИЙ СПИСОК}

1. Аврунев Е.И., Гиниятов А.И. Современное состояние и проблемы геодезического обеспечения создания и ведения трехмерного кадастра недвижимости [Электронный ресурс]: Регулирование земельно-имущественных отношений в России: правовое и геопространственное обеспечение, оценка недвижимости, экология, технологические решения: сб. материалов 3-й Национальной научно-практической конференции, 27-29 ноября 2019 г., Новосибирск, СГУГиТ.- Режим доступа: http://nir.sgugit.ru/elektronnye-publikatsii-noyab19/.

2. Создание модели трехмерного кадастра недвижимости в России. G2G10/RF/9/1.Заключительный отчет. [Электронный ресурс]- Режим доступа: https://portal.rosreestr.ru/wps/portal/cc_news?news_id=16202\&news_line_id=11662.

3. Natalia Vandysheva, Vladimir Tikhonov, Peter Van Oosterom, Jantien Stoter, Hendrik Ploger, Rik Wouters, Veliko Penkov 3D Cadastre Modelling in Russia, FIG Working Week 2011 Bridging the Gap between Cultures Marrakech, Morocco, 18-22 May 2011 [Электронный ресурс]Режим доступа: $\quad$ https://www.researchgate.net/publication/241886547_ 3D_Cadastre_modelling_in_Russia.

4. Чернов А. В. Разработка и исследование методики формирования трехмерного кадастра недвижимости: дисс. на соиск. учен. степ. канд. техн. наук по спец. 25.00.26. / А.В. Чернов // Новосибирск: СГУГиТ. - 2018. - 159 с.

5. Аврунев Е.И., Чернов А.В, Дубровский А.В., Комиссаров А.В., Пасечник Е.Ю. Технологические аспекты построения 3D- модели инженерных сооружений в городах Арктического региона РФ. Известия Томского политехнического университета. Инжиниринг георесурсов. - 2018. - Т. 329, № 7.131-137c.

6. Tor Valstad, Developments of the 3D Cadastre in Norway, XXIII FIG Congress 2006 [Электронный ресурс]- Режим доступа: http://www.gdmc.nl/3DCadastres/literature/ 3Dcad_2006_03.pdf.

7. Accuracy of 3D Building Models Created Using Terrestrial and Airborne Laser Scanning Data [Электронный ресурс] / A. Borkowski, G. Jozkow, M. Ziaja, K. Becek // FIG Congress 2014. Engaging the Challenges - Enhancing the Relevance. - Kuala Lumpur, Malaysia, 2014. - Режим доступа: http:/www.fig.net/resources/proceedings/ fig_proceedings/fig2014/papers/ ts08k/TS08K_borkowski_jozkow_et_al_7003_abs .pdf. - Загл. с экрана. 
8. Eriksson G., Adolfsson C. Experiences of the 3D Cadastre Legislation, XXIII FIG Congress 2006; Eriksson G., Jansson L. Strata titles are introduced in Sweden. - FIG International Congress, 2010: [Электронный ресурс]- Режим доступа: http://www.fig.net/pub/fig2010/papers/ts05a\%5Cts05a_eriksson_jansson_3909.pdf.

9. Peter van Oosterom, Jantien Stoter, Hendrik Ploeger, Christiaan Lemmen, Rod Thompson and Sudarshan Karki Initial Analysis of the Second FIG 3D Cadastres Questionnaire: Status in 2014 and Expectations for 2018, 4th International Workshop on 3D Cadastres, 9-11 November 2014, Dubai, United Arab Emirates [Электронный pecypc] - Режим доступа: http://www.gdmc.nl/publications/2014/Second_FIG_3D_Cadastres_Questionnaire.pdf.

10. Аврунев Е.И. Геодезическое обеспечение Государственного кадастра недвижимости [Текст] : монография / Е.И. Аврунев. - Новосибирск: СГГА, 2010. - 144 с.

11. Карпик А.П. Методологические и технологические основы геоинформационного обеспечения территорий: Монография. - Новосибирск: СГГА, 2004. - 260 с.

12. Карпик, А. П. Сущность геоинформационного пространства территорий как единой основы развития государственного кадастра недвижимости [Текст] / А. П. Карпик, В. С. Хорошилов // Изв. вузов. Геодезия и аэрофотосъемка. - 2012. - № 1. - С. 134-136.

13. Карпик А. П. Системная связь устойчивого развития территорий с его геодезическим информационным обеспечением // Вестник СГГА. - 2010. - Вып. 1 (12). - С. 3-11.

14. Метелева М. В.Разработка и исследование методики координатного обеспечения кадастровой деятельности в территориальных образованиях: дисс. на соиск. учен. степ. канд. техн. наук по спец. 25.00.26. / М.В. Метелева // Новосибирск: СГУГиТ. - 2015. - 150с.

15. ГОСТ 21779-82 (СТ СЭВ 2681-80) Система обеспечения точности геометрических параметров в строительстве. Технологические допуски. Переиздание. Май 1993 г.

(С Е. И. Аврунев, А. И. Гиниятов, 2021 